\title{
Optical pulsars and polarimetry
}

\author{
Andrew Shearer and Eoin O' Connor \\ Centre for Astronomy, School of Physics, NUI Galway \\ Galway, Ireland \\ email: andy.shearer@nuigalway.ie
}

\begin{abstract}
Despite the early optical detection of the Crab pulsar in 1969, optical pulsars have become the poor cousin of the neutron star family. Only five normal pulsars have been observed to pulse in the optical waveband. A further three magnetars/SGRs have been detected in the optical/near IR. Optical pulsars are intrinsically faint with a first order luminosity, predicted by Pacini, to be proportional to $\mathrm{P}^{-10}$, where $\mathrm{P}$ is the pulsar's period. Consequently they require both large telescopes, generally over-subscribed, and long exposure times, generally difficult to get. However optical observations have the benefit that polarisation and spectral observations are possible compared to X-ray and gamma-ray observations where polarisation measurements are limited. Over the next decade the number of optical pulsars should increase as optical detectors approach $100 \%$ quantum efficiency and as we move into the era of extremely large telescopes where limiting fluxes will be 30 to 100 times fainter compared to existing optical telescopes.
\end{abstract}

Keywords. stars: neutron, (stars:) pulsars: general, (stars:) pulsars: individual (Crab, Vela, Geminga, B0540-69, B0656+14), polarization

\section{Introduction}

Optical observations of pulsars had a promising start with the first observations of the Crab pulsar (Cocke et al. (1969)). The next optical pulsar, Vela, was identified in 1978 (Peterson et al. (1978)) and since then only five more optical pulsars have been identified; PSR B0540-69 (Middleditch et al. (1987)), PSR B0656+14 (Shearer et al. (1997), Kern et al. (2003)), Geminga (Shearer et al. (1998)) and very recently PSR $\mathrm{J} 1023+0038$ (Ambrosino et al. (2017)). The latter is the first millisecond pulsar with a pulsed optical counterpart. Table 1 shows the current number of pulsars with known optical counterparts. From Table 1 it is clear that optical pulsars are faint. Indeed Franco Pacini predicted that the luminosity would scale with the pulsar's period, $\mathrm{P}$, as $P^{-10}$ based on an incoherent synchrotron origin for the optical photons (Pacini (1971)). This has been confirmed to first order, although Pacini's initial proposal has to be modified for the effect of duty cycle (Pacini \& Salvati (1983)) and, in the future, presumably with a better understanding of each system's geometry.

As the expected emission mechanism for the optical radiation is an incoherent synchrotron process it is expected that the radiation will be polarised. Early observations of the Crab pulsar (Cocke et al. (1970)) confirmed this. Table 1 summarises the current number of optical pulsars with their polarisation measurement where appropriate. Note there are over 2,500 radio pulsars, but only 6 have measured pulsed optical emission. From Iqueye observations of the Crab pulsar individual optical pulses do not show marked pulse - pulse variability in contrast to pulsar radio emission (Naletto et al. (2009) ). Two correlations have been observed between radio emisson and optical emission. Firstly, during giant radio pulse (GRP) events the optical emission is enhanced by $\approx 3 \%$, Shearer et al. (2003), Strader et al. (2013), see Fig. 3 This enhancement is also seen to increase when the GRP coincides with the peak of the optical pulse, indicative of a similar emission zone for both radio and optical photons, Shearer et al. (2012), Strader et al. (2013). 

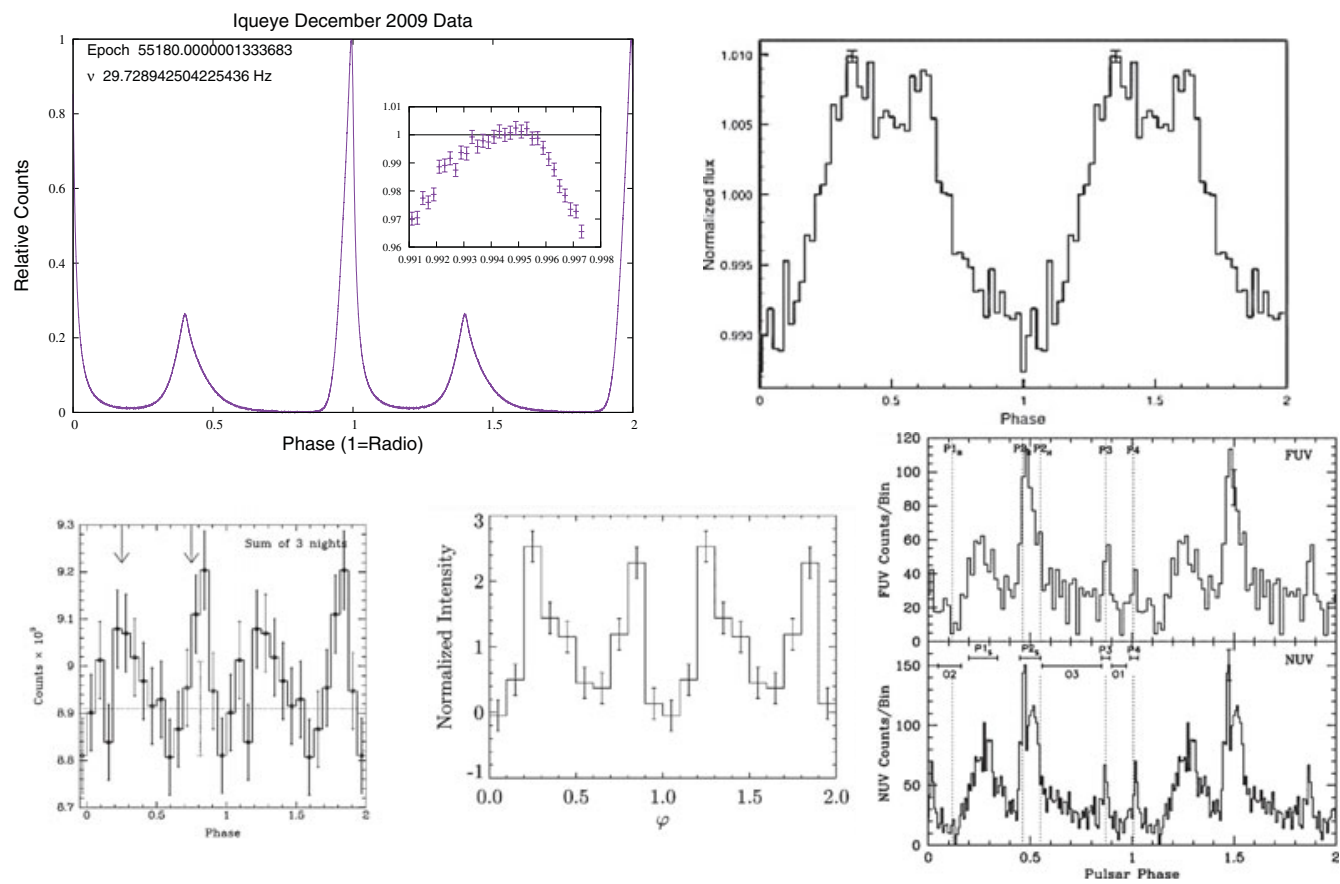

Figure 1. Light curves from the known optical pulsars. Top left Crab (Collins et al. (2012)); Top right PSR B0540-69 (Gradari et al. (2011)); Bottom left Geminga (Shearer et al. (1998)); middle PSR B0656+14 (Kern et al. (2003)); right Vela (Romani et al. (2005)). The Crab pulsar's optical peak is at phase 0.9947 corresponding to $178 \mu \mathrm{sec}$ before the radio pulse. The peak appears flat within the statistical uncertainties for about $40 \mu \mathrm{sec}$.
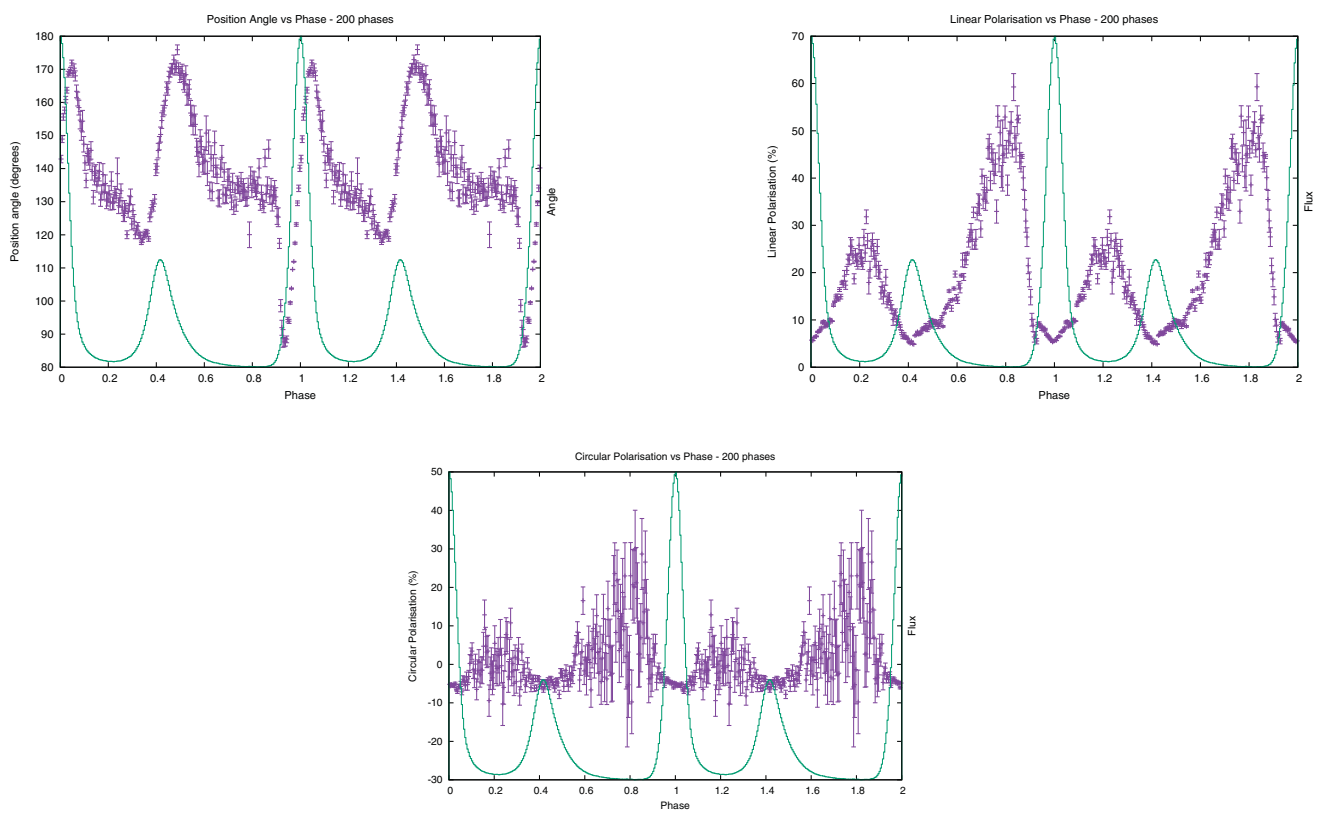

Figure 2. GASP (Collins et al. (2013)) polarisation measurements of the Crab pulsar taken on the $4.2 \mathrm{~m}$ WHT in December 2015: angle (left), degree (middle) and circular (right). Each data point is equivalent to about 50 seconds of observations from 10,000 seconds observations and 200 phase bins. Each error bar represents $1 \sigma$ statistical fluctuations. 


\begin{tabular}{|c|c|c|c|c|c|c|c|c|c|}
\hline Name & Age & mag & $\begin{array}{l}\text { distance } \\
(\mathrm{kpc})\end{array}$ & $A_{V}$ & $\lambda$ & Pulsed & $(\%)$ & $\begin{array}{r}\text { arisation } \\
\left({ }^{\circ}\right)\end{array}$ & Reference \\
\hline Crab & 3.10 & 16.6 & 1.73 & 1.6 & $\mathrm{nUV}, \mathrm{O}, \mathrm{nIR}$ & $\mathrm{Y}$ & $9.7 \% \pm 0.1 \%$ & $139.8^{\circ} \pm 0.2^{\circ}$ & {$[1][2]$} \\
\hline Crab (PA) & & & & & & & $5.2 \% \pm 0.3 \%$ & $105.1^{\circ} \pm 1.6^{\circ}$ & [3] \\
\hline B $1509-58$ (PA) & 3.19 & $25.7^{R}$ & 4.18 & 5.2 & $\mathrm{O}$ & & $\sim 10.4 \%$ (no error) & & {$[4]$} \\
\hline В $0540-69$ & 3.22 & 22.0 & 49.4 & 0.6 & $\mathrm{O}$ & $\mathrm{Y}$ & $<15 \%$ & & {$[5]$} \\
\hline B 0540-69 (PA) & & & & & & & $\approx 5 \%$ (no error) & & [4] \\
\hline Vela $[\mathrm{PA}]$ & 4.05 & 23.6 & 0.23 & 0.2 & $\mathrm{nUV}, \mathrm{O}, \mathrm{nIR}$ & Y & $8.1 \% \pm 0.7 \%$ & $146.3^{\circ} \pm 2.4^{\circ}$ & {$[6]$} \\
\hline Geminga & 5.53 & 25.5 & 0.07 & 0.07 & $\mathrm{nUV}, \mathrm{O}, \mathrm{nIR}$ & $\mathrm{Y}$ & & & \\
\hline В $0656+14$ & 5.05 & 25.0 & 0.29 & 0.09 & $\mathrm{nUV}, \mathrm{O}, \mathrm{nIR}$ & Y & $100 \%$ & $135^{\circ} \pm 20^{\circ}$ & [7] \\
\hline B $0656+14(\mathrm{PA})$ & & & & & & & $11.9 \% \pm 5.5 \%$ & $126^{\circ} \pm 13^{\circ}$ & [8] \\
\hline $\mathrm{J} 1023+0038$ & 9.4 & $22(?)$ & 1.4 & & $\mathrm{O}$ & $\mathrm{Y}$ & & & \\
\hline B $1055-52$ & 5.73 & $24.9^{U}$ & 0.72 & 0.22 & $\mathrm{nUV}, \mathrm{O}$ & & & & \\
\hline B $1929+10$ & 6.49 & $25.6^{U}$ & 0.33 & 0.15 & $\mathrm{nUV}$ & & & & \\
\hline B $1133+16$ & 6.69 & 28 & 0.35 & 0.12 & $\mathrm{O}$ & & & & \\
\hline B $0950+08$ & 7.24 & 27.1 & 0.26 & 0.03 & $\mathrm{nUV}, \mathrm{O}$ & & & & \\
\hline J0108-1431 & 8.3 & $26.4^{U}$ & 0.2 & 0.03 & $\mathrm{O}$ & & & & \\
\hline $\mathrm{J} 0437-4715$ & 9.20 & & 0.14 & 0.11 & $n U V$ & $\mathrm{Y}$ & & & \\
\hline
\end{tabular}

(PA) - polarisation refers to phase averaged polarisation, other values refer to the peak of the phase resolved polarisation. [1] Słowikowska et al. (2009), this work; [2] Smith et al. (1988); [3] Moran et al. (2013); [4] Wagner \& Seifert (2000), [5] Middleditch et al. (1987); [6] Moran et al. (2014), [7] Kern et al. (2003), [8] Mignani et al. (2015)

Table 1. Normal pulsars with detected optical emission, based on Mignani (2011).
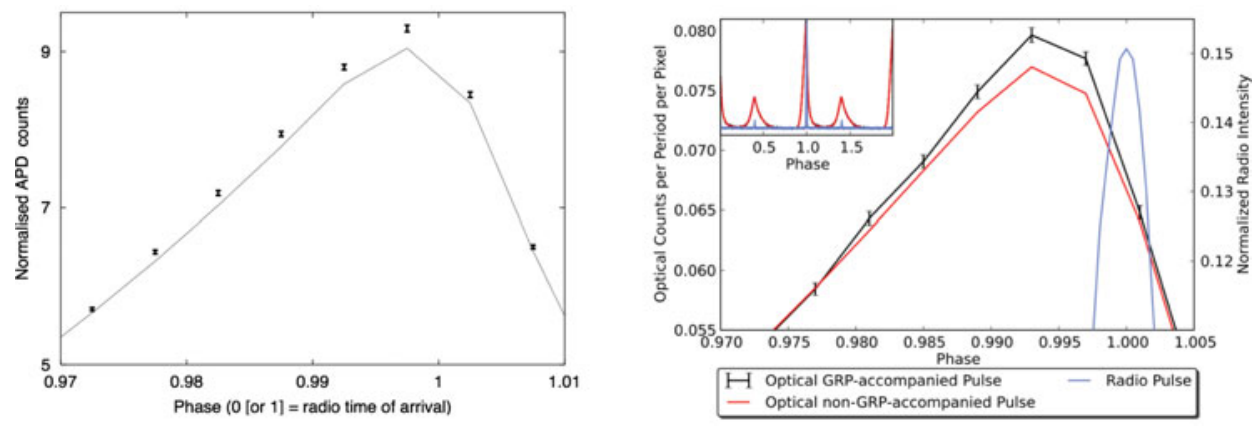

Figure 3. Optical enhancement during GRP events. Left : Shearer et al. (2003); Right Strader et al. (2013)

Secondly, the peak of the optical linear polarisation coincides with the radio precursor pulsar, Słowikowska et al. (2009), this correlation has no definitive explanation.

Optical pulsar studies are restricted by their extreme faintness. It is likely that future detections will have visual magnitudes greater than 27, corresponding to a few nJy. Such faint sources require large $(>30 \mathrm{~m})$ optical telescopes which are due to see first light in the next decade. In particular Micado (Davies et al. (2016)), on the European Extremely Large Telescope, will have a limiting J band magnitude of about 30 with a $0.04 \mathrm{sec}$ time resolution after an 1 hour integration, making it suitable for magnetar and slower pulsar observations. It is hoped that higher time resolution systems will be available for the second tranche of instruments. Detector possibilities include microwave kinetic induction devices (MKIDs) Strader et al. (2013) and e-Avalanche Photodiode (e-APD) arrays (Finger et al. (2016)). Polarimetric observations of the known optical pulsars are planned using the Galway Astronomical Stokes Polarimeter (GASP), once these are complete new instruments and larger optical telescopes will be needed for the fainter pulsars. 


\section{Acknowledgements}

EOC wishes to acknowledge the support of the Irish Research Council under project funding GOIPG/2014/1266. We also acknowledge Science Federation of Ireland (SFI) for funding under the SFI Strategic Opportunity Fund for Research Infrastructure, This research was made possible by telescope time made available through the the European Community's Seventh Framework Programme (FP7/2013-2016) Opticon ( 312430) Transnational Access programme.

\section{References}

Ambrosino, F., Papitto, A., Stella, L., et al. 2017, Nature Astronomy, 1, 266

Cocke, W. J., Disney, M. J., \& Muncaster, G. W. 1970, Nature, 227, 1327

Cocke, W. J., Disney, M. J., \& Taylor, D. J. 1969, Nature, 221, 525

Collins, P., Kyne, G., Lara, D., et al. 2013, Experimental Astronomy, 36, 479

Collins, S., et al. 2012, New Horizons in Time Domain Astronomy, 285, 296

Davies, R., Schubert, J., Hartl, M., et al. 2016, SPIE, 9908, 99081Z

Finger, G., Baker, I., Alvarez, D., et al. 2016, SPIE, 9909, 990912

Gradari, S., Barbieri, M., Barbieri, C., et al. 2011, MNRAS, 412, 2689

Kern, B., Martin, C., Mazin, B., \& Halpern, J. P. 2003, ApJ, 597, 1049

Middleditch, J., Pennypacker, C. R., \& Burns, M. S. 1987, ApJ, 315, 142

Mignani, R. P., Moran, P., Shearer, A., et al. 2015, A\&A, 583, A105

Mignani, R. P. 2011, Advances in Space Research, 47, 1281

Moran, P., Shearer, A., Mignani, R. P., et al. 2013, MNRAS, 433, 2564

Moran, P., Mignani, R. P., \& Shearer, A. 2014, MNRAS, 445, 835

Naletto, G., Barbieri, C., Occhipinti, T., et al. 2009, A\&A, 508, 531

Pacini, F. 1971, ApJ (Letters), 163, L17

Pacini, F. \& Salvati, M. 1983, ApJ, 274, 369

Peterson, B. A., Murdin, P., Wallace, P., et al. 1978, Nature, 276, 475

Romani, R. W., Kargaltsev, O., \& Pavlov, G. G. 2005, ApJ, 627, 383

Shearer, A., et al., 2012, Electromagnetic Radiation from Pulsars and Magnetars, 466, 11

Shearer, A., 2008, Astrophysics and Space Science Library, 351, 1

Shearer, A., Stappers, B., O'Connor, P., et al., 2003, Science, 301, 493

Shearer, A., Golden, A., Harfst, S., et al. 1998, A\&A, 335, L21

Shearer, A., Redfern, R. M., Gorman, G., et al. 1997, ApJ (Letters), 487, L181

Słowikowska, A., Kanbach, G., Kramer, M., \& Stefanescu, A. 2009, MNRAS, 397, 103

Smith, F. G., Jones, D. H. P., Dick, J. S. B., \& Pike, C. D. 1988, MNRAS, 233, 305

Strader, M. J., Johnson, M. D., Mazin, B. A., et al. 2013, ApJ (Letters), 779, L12

Wagner, S. J. \& Seifert, W. 2000, IAU Col. 177: Pulsar Astronomy - 2000 and Beyond, 202, 315 